Published in final edited form as:

Dev Neuropsychol. 2015 January ; 40(1): 40-44. doi:10.1080/87565641.2014.979927.

\title{
Decreased Cerebral Blood Flow in Chronic Pediatric Mild TBI: An MRI Perfusion Study
}

\section{Yang Wang,}

Department of Radiology, Medical College of Wisconsin, Milwaukee, Wisconsin, and Center for Neuroimaging, Department of Radiology and Imaging Sciences, Indiana University School of Medicine, Indianapolis, Indiana

John D. West,

Center for Neuroimaging, Department of Radiology and Imaging Sciences, Indiana University School of Medicine, Indianapolis, Indiana

Jessica N. Bailey,

Center for Neuroimaging, Department of Radiology and Imaging Sciences, Indiana University School of Medicine, Indianapolis, Indiana

\section{Daniel R. Westfall,}

Center for Neuroimaging, Department of Radiology and Imaging Sciences, Indiana University School of Medicine, Indianapolis, Indiana, and Neuroscience Program, University of Illinois at Urbana-Champaign, Urbana, Illinois

Hui Xiao,

Center for Neuroimaging, Department of Radiology and Imaging Sciences, Indiana University School of Medicine, Indianapolis, Indiana, and Department of Radiology, Dongfang Hospital, Fujian, China

\section{Todd W. Arnold,}

St.Vincent Sports Performance, Carmel, Indiana

Patrick A. Kersey,

St.Vincent Sports Performance, Carmel, Indiana

Andrew J. Saykin, and

Center for Neuroimaging, Department of Radiology and Imaging Sciences, Indiana University School of Medicine, Indianapolis, Indiana

\section{Brenna C. McDonald}

Center for Neuroimaging, Department of Radiology and Imaging Sciences, Indiana University School of Medicine, Indianapolis, Indiana

\section{Abstract}

\footnotetext{
Copyright $@ 2015$ Taylor \& Francis Group, LLC

Correspondence should be addressed to Yang Wang, M.D., Associate Professor, Department of Radiology, Center for Imaging Research, Department of Biophysics, Medical College of Wisconsin, 9200 West Wisconsin Avenue, MFRC, G2411, Milwaukee, WI 53226. yangwang@mcw.edu.

Color versions of one or more of the figures in the article can be found online at www.tandfonline.com/hdvn.
} 
We evaluated cerebral blood flow (CBF) in chronic pediatric mild traumatic brain injury (mTBI) using arterial spin labeling (ASL) magnetic resonance imaging perfusion. mTBI patients showed lower CBF than controls in bilateral frontotemporal regions, with no between-group cognitive differences. Findings suggest ASL may be useful to assess functional abnormalities in pediatric mTBI.

Pediatric mild traumatic brain injury (mTBI) or concussion is a significant public health concern. While symptoms typically resolve, persistent sequelae from mild injuries in some patients can be particularly disruptive to the developing brain, and may be longer-lasting in children, potentially contributing to the development of further emotional, behavioral, and cognitive concerns (Choe, Babikian, DiFiori, Hovda, \& Giza, 2012; Maugans, Farley, Altaye, Leach, \& Cecil, 2012). The developing brain differs from that of a mature adult, as neuronal systems develop at different rates during childhood and brain changes are dramatic. Thus, the injury and recovery processes may also differ, especially while still symptomatic from an initial injury (Choe et al., 2012). Delayed recovery of cognitive function and other self-reported symptoms have been found in children compared with adults after concussion. This lengthened recovery may place youth at risk for repeated concussions. Even after symptom resolution, neurons under such a state of physiologic stress function abnormally and may remain susceptible to second injury (Maugans et al., 2012).

The pathophysiology of concussion is poorly understood. It is increasingly clear that cerebrovascular alterations play a role in the evolution of TBI sequelae as well as in the process of brain repair (Len \& Neary, 2011; Metting, Spikman, Rödiger, \& van der Naalt, 2014). Measurement of cerebral blood flow (CBF) in vivo can therefore enhance the understanding of neurovascular mechanisms underpinning recovery from TBI, and how pediatric injury may alter neuronal functioning and contribute to persistent sequelae. Arterial spin labeling (ASL) is an advanced magnetic resonance imaging (MRI) technique that measures CBF noninvasively using magnetically labeled arterial blood water as an endogenous contrast tracer, without radiation exposure (Wang et al., 2003). This study examined CBF in chronic pediatric mTBI patients compared to healthy controls (HC). We hypothesized that mTBI patients would demonstrate lower regional CBF than HC.

\section{METHODS}

Participants included 14 adolescents (mean age $15.1 \pm 0.92$ years) who sustained a concussion/mTBI 3-12 months (mean 7.4 \pm 2.4 ) prior to participation. Mechanism of injury was sports-related/recreational; seven patients reported prior concussions. The HC group included 15 adolescents (mean age $14.5 \pm 0.99$ years) with no history of TBI. All participants had no history of other neurological, psychiatric, or systemic medical disorder likely to affect brain function.

A targeted neuropsychological and parent-report battery focused on attention-related and executive functions and episodic memory, given the high incidence of subjective complaints in these areas following mTBI and prior research suggesting that measures in these domains are most sensitive to concussion-related changes (McCrea et al., 2009). Screening measures for intellect, academic achievement, language, psychomotor skills, and affective/behavioral 
status were also administered. Subtest scores were z-transformed based on the HC group mean, combined into cognitive domain scores (memory, executive functioning, attention, processing and motor speed, and reaction time), and analyzed using SPSS (version 21).

MRI was conducted on a 3T scanner (Siemens Tim Trio) using a 12-channel head coil. Subjects were scanned in a conscious resting state with closed eyes. Cerebral perfusion measurements used the Q2TIPS PASL sequence (Wang et al., 2011). Optimized inversion time delays were chosen as $\mathrm{TI}_{1} / \mathrm{TI}_{2}=700 / 1800 \mathrm{~ms}$ to minimize intravascular signal intensity. Interleaved label and control images were acquired using a gradient-echo single shot EPI readout, with parameters: TR/TE $=3000 / 13 \mathrm{msec}, 24$ axial slices with voxel size of 3.75 $\mathrm{mm} \times 3.75 \mathrm{~mm} \times 5 \mathrm{~mm}$. Each PASL scan has 100 dynamic measurements (50 control and label image pairs) plus one $\mathrm{M}_{0}$ map. Online 3D motion correction was applied to minimize head motion artifact during acquisition. A high resolution T1-weighted MPRAGE was acquired for anatomical reference, and an SWI series was obtained for neuroradiological read.

PASL data analysis was performed using an updated version of our previously published methods (Wang et al., 2011). Images were corrected for motion, pair-wise subtracted between label and control images, and averaged to generate a mean difference image. Quantitative CBF maps were calculated based on a single compartment model incorporating in vivo measurement of blood $\mathrm{T}_{1}$ and labeling efficiency (Wang et al., 2003, 2011). Individual CBF maps were co-registered with the MPRAGE, then normalized using the canonical Montréal Neurological Institute template and spatially smoothed. The general linear model including age and sex as covariates was utilized in SPM8 for voxel-wise group analysis, with Family-Wise Error (FWE) correction for multiple comparisons. Mean CBF was compared in SPSS.

\section{RESULTS}

Clinical neuroradiological reading revealed no acute intracranial abnormalities. There were no between-group (HC versus mTBI) differences for age, gender, maternal education, or any neuropsychological domains. mTBI patients showed significantly lower CBF than $\mathrm{HC}$ in bilateral frontotemporal regions (overall $\mathrm{p}_{\text {crit }}<.01$, cluster-level $\mathrm{p}_{\text {FWE-corr }}<.05$, Figure 1 ). No region showed significantly greater CBF in mTBI patients than HC. mTBI groups (1 mTBI versus $>1 \mathrm{mTBI})$ did not differ in mean $\mathrm{CBF}(p=.359)$, although both showed lower $\mathrm{CBF}$ than $\mathrm{HC}(p s=.022$ and .002 , respectively, Figure 1). mTBI groups did not differ from each other or $\mathrm{HC}$ for neuropsychological performance.

\section{DISCUSSION}

We found lower regional CBF in pediatric mTBI patients on average seven months postinjury, despite normal conventional MRI and neuropsychological performance. These findings are consistent with work by Maugans and colleagues (2012) using MR phase contrast angiography showing reduced $\mathrm{CBF}$ after pediatric concussion which persisted $>30$ days post-injury. No prior studies have utilized PASL to examine CBF in pediatric mTBI. It has been theorized that pediatric concussion may be primarily a neurophysiologic injury, 
affecting CBF without evidence of measurable structural, metabolic, neuronal, or axonal injury (Maugans et al., 2012). Our results suggest that pediatric concussion may produce a pathophysiologic process resulting in altered CBF, with a variable and possibly protracted timeframe for resolution. Understanding the pathophysiology of pediatric concussion may open novel therapeutic avenues to decrease symptom persistence and prevent further injury.

Traditional brain imaging or cognitive assessment techniques appear insensitive to underlying pathophysiologic post-concussive processes; advanced neuroimaging applications may provide more specificity in accurately diagnosing mTBI. ASL perfusion measurement appears well-suited for longitudinal studies of CBF in healthy and diseased individuals, or as a surrogate marker of metabolism (Detre, Rao, Wang, Chen, \& Wang, 2012; Wang et al., 2003). Very limited applications of ASL in adult patients with chronic TBI have demonstrated decreased CBF (Ge et al., 2009; Kim et al., 2010). Extending these previous findings, our results also suggest that ASL MRI may serve as an important tool to assess underlying functional abnormality in adolescents with chronic mTBI. One limitation of the present study is a modest sample size, limiting ability to examine the relationship of $\mathrm{CBF}$ to other factors of interest such as cognitive or injury variables. For example, we compared mTBI patients with one versus multiple concussions, and found no between-group differences in mean CBF, though both were reduced relative to HC. Graphical illustration of the data, however, suggest that with larger samples this effect may become significant. Such a finding could have important clinical implications in terms of risk of subsequent concussion. Larger longitudinal studies should assess CBF changes at different post-injury stages and relate findings to cognitive and post-concussive symptom status, to further elucidate mechanisms underlying brain dysfunction and recovery in pediatric mTBI. Comparison and combination with other advanced neuroimaging modalities will be critical to generate imaging biomarkers to guide targeted treatment and management approaches in the future.

\title{
Acknowledgments
}

\author{
FUNDING
}

This work was supported by a grant from the Indiana State Department of Health Spinal Cord and Brain Injury Fund Research Grant Program and by a Project Development Team within the ICTSI NIH/NCRR Grant Number RR025761.

\section{References}

Choe MC, Babikian T, DiFiori J, Hovda DA, Giza CC. A pediatric perspective on concussion pathophysiology. Current Opinion in Pediatrics. 2012; 24(6):689-695.10.1097/MOP. 0b013e32835a1a44 [PubMed: 23080130]

Detre JA, Rao H, Wang DJ, Chen YF, Wang Z. Applications of arterial spin labeled MRI in the brain. Journal of Magnetic Resonance Imaging. 2012; 35(5):1026-1037.10.1002/jmri.23581 [PubMed: 22246782]

Ge Y, Patel MB, Chen Q, Grossman EJ, Zhang K, Miles L, Grossman RI. Assessment of thalamic perfusion in patients with mild traumatic brain injury by true FISP arterial spin labelling MR imaging at 3T. Brain Injury. 2009; 23(7):666-674.10.1080/02699050903014899 [PubMed: 19557570] 
Kim J, Whyte J, Patel S, Avants B, Europa E, Wang J, Detre JA. Resting cerebral blood flow alterations in chronic traumatic brain injury: An arterial spin labeling perfusion FMRI study. Journal of Neurotrauma. 2010; 27(8):1399-1411.10.1089/neu.2009.1215 [PubMed: 20528163]

Len TK, Neary JP. Cerebrovascular pathophysiology following mild traumatic brain injury. Clinical Physiology and Functional Imaging. 2011; 31(2):85-93.10.1111/j.1475-097X.2010.00990.x [PubMed: 21078064]

Maugans TA, Farley C, Altaye M, Leach J, Cecil KM. Pediatric sports-related concussion produces cerebral blood flow alterations. Pediatrics. 2012; 129(1):28-37.10.1542/peds.2011-2083 [PubMed: 22129537]

McCrea M, Iverson GL, McAllister TW, Hammeke TA, Powell MR, Barr WB, Kelly JP. An integrated review of recovery after mild traumatic brain injury (MTBI): Implications for clinical management. Clinical Neuropsychology. 2009; 23(8):1368-1390.10.1080/13854040903074652

Metting Z, Spikman JM, Rödiger LA, van der Naalt J. Cerebral perfusion and neuropsychological follow up in mild traumatic brain injury: Acute versus chronic disturbances? Brain Cognition. 2014; 86:24-31.10.1016/j.bandc.2014.01.012 [PubMed: 24556319]

Wang J, Licht DJ, Jahng GH, Liu CS, Rubin JT, Haselgrove J, Detre JA. Pediatric perfusion imaging using pulsed arterial spin labeling. Journal of Magnetic Resonance Imaging. 2003; 18(4):404413.10.1002/jmri.10372 [PubMed: 14508776]

Wang Y, Saykin AJ, Pfeuffer J, Lin C, Mosier KM, Shen L, Hutchins GD. Regional reproducibility of pulsed arterial spin labeling perfusion imaging at 3T. Neuroimage. 2011; 54(2):11881195.10.1016/j.neuroimage.2010.08.043 [PubMed: 20800097] 


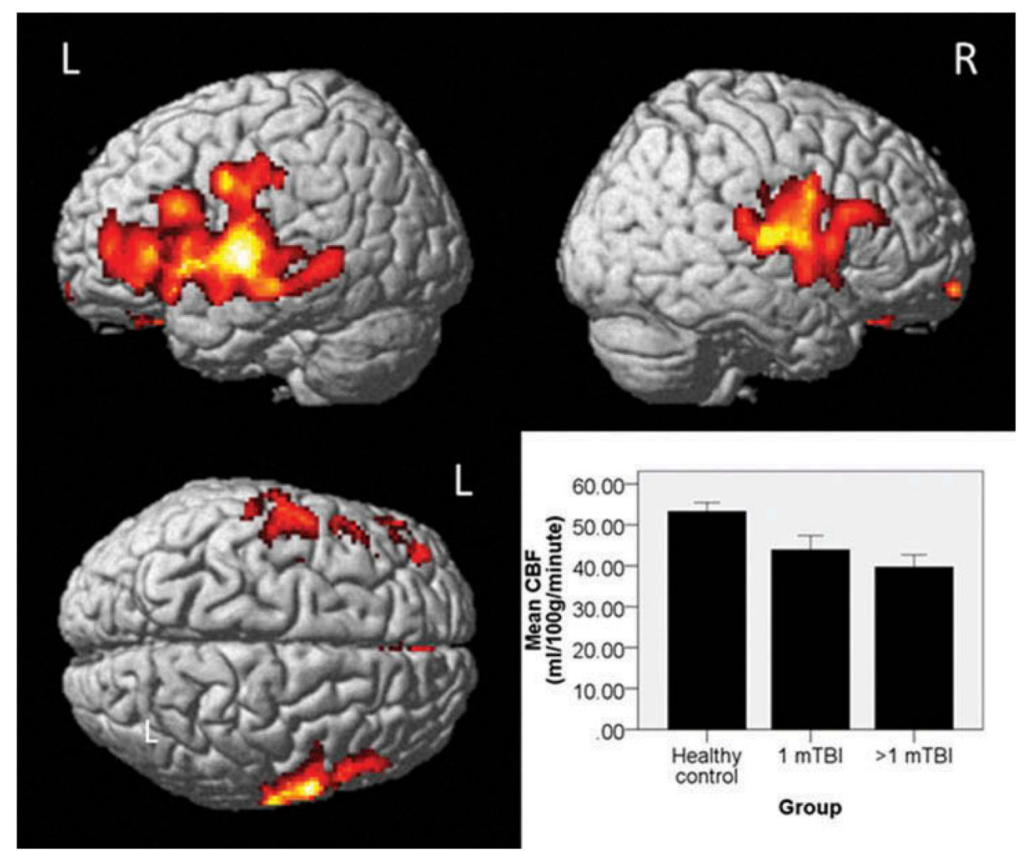

FIGURE 1.

Between-group differences in cerebral perfusion as measured by PASL (Pulsed Arterial Spin Labeling) magnetic resonance imagining. Healthy controls showed significantly greater cerebral blood flow (CBF) than mild traumatic brain injury (mTBI) patients in bilateral frontotemporal regions. No regions showed significantly greater CBF in mTBI patients than controls. There was no significant difference in mean CBF between patients who had a history of 1 versus $>1 \mathrm{mTBI}$, although both mTBI groups showed lower CBF than controls (see text for $p$ values, error bars are standard error, $\mathrm{L}=\mathrm{Left}, \mathrm{R}=\mathrm{Right}$ ). 